\title{
Problematização como método ativo de ensino-aprendizagem em um Curso de Odontologia
}

Rodrigo Guerra de Oliveira*, André Luiz Dias**, Antônio Márcio Lima Ferraz Júnior**, Fernanda Ribeiro Porto**, Fernando Luiz Hespanhol**, Rinaldo Henrique Aguilar da Silva***, Djalma Rabelo Ricardo****

\author{
* Doutor, Professor e Coordenador do Curso de \\ Odontologia da Faculdade de Ciências Médicas e da \\ Saúde de Juiz de Fora (SUPREMA). \\ ** Mestre, Professor do Curso de Odontologia da Faculdade \\ de Ciências Médicas e da Saúde de Juiz de Fora \\ (SUPREMA). \\ *** Doutor, Professor da Faculdade de Medicina de Marília \\ (FAMEMA). \\ **** Doutor; Diretor de Ensino, Pesquisa e Extensão da \\ Faculdade de Ciências Médicas e da Saúde de Juiz de \\ Fora (SUPREMA).
}

\section{RESUMO}

As Diretrizes Curriculares Nacionais para os cursos de Odontologia lançadas no ano de 2002 indicaram uma concepção mais ampla de saúde e apontaram como necessidade um papel ativo dos estudantes no processo de ensino-aprendizagem, propondo a mudança da ênfase nos conteúdos para a participação mais ativa e independente e a superação da dicotomia entre teoria e prática, valorizando o trabalho articulado com os serviços de saúde. O presente estudo tem por objetivo verificar o efeito da problematizaçao sobre o desempenho na construção das questões de apreendizagem em funçao da área do conhecimento, eixos de competência e taxonomia de Bloom das questões. Foram avaliados 93 estudantes ao longo de quatro semestres. A partir das experiências vivenciadas na clínica, durante o atendimento a pacientes, foram levantadas lacunas de conhecimento no ciclo pedagógico, onde se aplicou a metodologia da problematização. Seguindo os passos tutoriais, foram registradas 182 questões. Dessas, 67 que não se repetiram foram agrupadas por área do conhecimento $(67,2 \%$ de abordagem cognitiva, $25,3 \%$ psicomotora e $7,5 \%$ afetiva). De acordo com a taxonomia de Bloom, 32 questões foram consideradas de baixa taxonomia, 22 de média e 13 de alta taxonomia. A partir dos resultados encontrados no presente estudo pode-se concluir que, ao aplicar a problematização como método ativo de ensino-aprendizagem surgiram, na maioria, questões de abordagem cognitiva e de baixa taxonomia de Bloom.

Descritores: Aprendizagem Baseada em Problemas. Educação em Saúde. Avaliação educacional.

\section{INTRODUÇÃ̃O}

A Problematização como método ativo de ensino-aprendizagem possibilita a construção dos conhecimentos a partir dos problemas da realidade, bem como a integração de conteúdos básicos e profissionalizantes e entre a teoria e a prática 
para se superar os limites da formação e das práticas clínicas tradicionais ${ }^{1}$. O objetivo de construir conhecimentos a partir da realidade vivida pelos estudantes durante o processo de trabalho faz com que tal método favoreça o desenvolvimento de competência profis-sional. Posibilita impor a revisão dos conteúdos e da orientação pedagógica no Curso de Odontologia. Desta forma, visa atender a orientação das novas Diretrizes Curriculares Nacionais ${ }^{2}$ que apontam para a formação de profissionais críticos, capazes de levar em conta a realidade social para prestar assistência humana e de qualidade, buscando formar agentes capazes de desenvolver atenção integral à saúde ${ }^{3}$. O Curso de Odontologia deve apresentar um projeto pedagógico construído coletivamente, centrado no estudante como sujeito de aprendizado e apoiado no professor como facilitador e mediador do processo ensino-aprendizagem ${ }^{2}$.

Considerando que a maioria dos estudantes aprende por meio de modelos tradicionais, como esperar que eles, na sua atuação profissional, possam agir de maneira diferente ${ }^{4}$. Esse paradigma clássico dominou nossa cultura durante centenas de anos, ao longo dos quais modelou a sociedade ocidental e, na saúde em geral, predomina até os dias de hoje. A influência deste paradigma sobre as ciências da saúde resultou no chamado modelo biomédico que constitui o alicerce conceitual da ciência moderna ${ }^{5}$.

No Curso de Odontologia da Faculdade de Ciências Médicas e da Saúde de Juiz de Fora (SUPREMA), essa mudança de paradigma está alicerçada na vivência do método pedagógico da problematização, segundo Bordenave e
Pereira $(1991)^{6}$. A problematização se dá em cinco etapas, articuladas entre si e que possibilitam que o estudante possa desenvolver a capacidade de aprender a aprender, a aplicar o conhecimento e a trabalhar em equipe. A Figura 1 demonstra como se dá essa articulação, denominada "Ciclo Pedagógico" na Instituição.

Benjamin Bloom é um dos autores mais citados nos trabalhos referentes à formulação de objetivos educacionais. A principal contribuição ao estudo do tema é a taxonomia dos objetivos educacionais ${ }^{7}$, em que se discutiu a necessidade e conveniência do estabelecimento de um quadro teórico de referência que facilitasse a comunicação entre pesquiadores desta temática. Desenvolveu-se um sistema de classificação para três domínios: cognitivo, afetivo e psicomotor ${ }^{8}$. A taxonomia de Bloom estimula o processo de educação continuada e ressalta sua importância na elaboração das estratégias educacionais ${ }^{9}$.

O presente estudo têve como objetivo verificar o efeito da problematização sobre o desempenho na construção das questões de apreendizagem, em funçao da área do conhecimento, eixos de competência e taxonomia de Bloom das questões.

\section{MÉTODOS}

Este trabalho foi aprovado pelo Comitê de Ética e Pesquisa da Faculdade de Ciências Médicas e da Saúde de Juiz de Fora - SUPREMA, sob o parecer número 313-11. Foram avaliados 93 estudantes, durante os Ciclos Pedagógicos realizados na disciplina Clínica Integrada Plena II, do $8^{\circ}$ período do Curso de Odontologia da Faculdade de Ciências Médicas e da Saúde de Juiz de Fora - SUPREMA. 


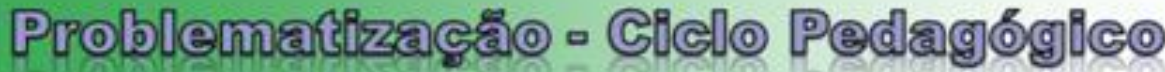

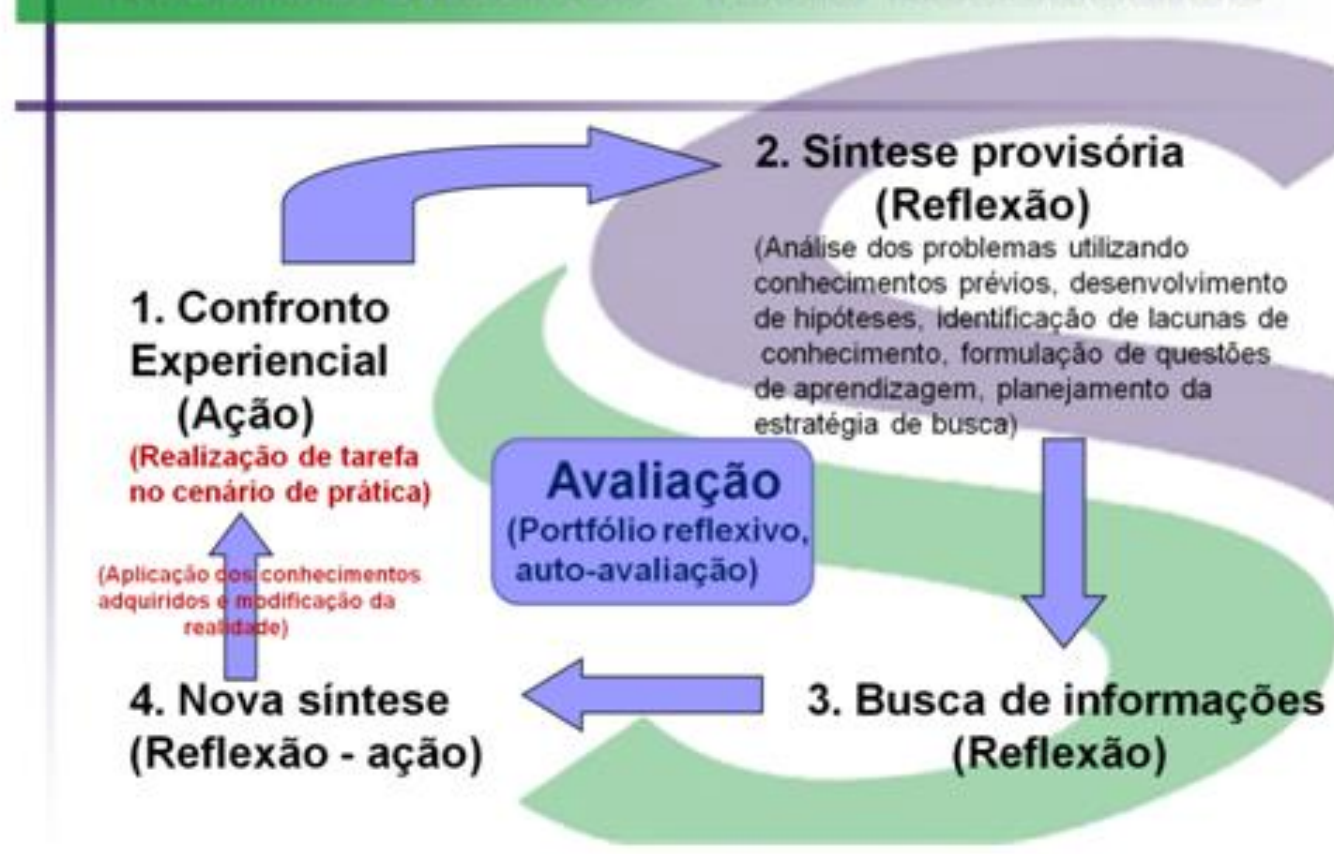

Figura 1. Etapas do ciclo pedagógico aplicado aos estudantes do curso de Odontologia da FCMS/JF - SUPREMA

A problematização é realizada em cinco etapas: 1) observação da realidade é a realização pelos estudantes da tarefa social e concreta, com base em um tema ou unidade de estudo; 2) pontos-chave - os estudantes são levados a refletir primeiramente sobre as possíveis causas da existência do problema em estudo; teorização - estudo, 3) investigação propriamente dita (os estudantes se organizam tecnicamente para buscar as informações de que necessitam sobre o problema onde quer que eles se encontrem, dentro de cada ponto-chave já definido); 4) hipóteses de solução - todo o estudo realizado deverá fornecer elementos para os estudantes, crítica e criativamente, elaborarem as possíveis soluções; 5) aplicação à realidade - são discutidas e encaminhadas à equipe de saúde as estratégias de intervenção ${ }^{4}$.

Os grupos formados por cerca de dez estudantes foram aleatoriamente divididos no início de cada semestre, sendo designado um preceptor para cada grupo. Os preceptores foram devidamente capacitados pelo Projeto de Capacitação Docente do Programa de Educação Continuada da Faculdade. As questões surgiram à partir das lacunas de conhecimento nas experiências vivenciadas na disciplina, durante $\mathrm{o}$ atendimento a pacientes, seguindo os passos tutoriais. Nos quatro semestres foram levantadas 182 questões, sendo registradas 67 questões distintas, agrupadas de acordo com a área do conhecimento e classificadas pela 
taxonomia do domínio cognitivo (Taxonomia de Bloom) por dois avaliadores distintos e previamente treinados. As questões discordantes foram submetidas à análise de um terceiro avaliador ${ }^{10}$.

As reuniões do ciclo pedagógico aconteciam semanalmente às quintas-feiras de $16 \mathrm{~h}$ às $17 \mathrm{~h} 40 \mathrm{~min}$ nas salas de aula da Faculdade reservadas para esta atividade. A frequência foi registrada no diário de classe online, assim como a etapa e conteúdo resumido. O registro acadêmico é informatizado e permite acesso rápido aos dados ao mesmo tempo que os documentam, através do sistema Totvs ${ }^{\circledR}$, em conformidade com o ministério da educação.

Após a apresentação do caso por um estudante, surgiam as lacunas de conhecimento. Estas eram anotadas por um estudante em uma coluna no quadro, conforme eram levantadas. As dúvidas quanto ao significado de palavras e termos eram esclarecidas. Em outra coluna no quadro eram anotadas as questões formuladas, que poderiam preencher/ solucionar as lacunas de conhecimento.
As questões eram revistas para não ficarem repetitivas e atenderem às demandas das lacunas de conhecimento.

Um formulário próprio era usado pelo tutor para registrar as lacunas de conhecimento e as questões que foram anotadas no quadro durante o cliclos pedagógicos.

\section{RESULTADOS}

Ao longo do período avaliado foram levantadas 182 questões. Do total registrado, as 67 que não se repetiram foram agrupadas por área do conhecimento, como descrito na Figura 2.

Do total, 67,2\% foram de abordagem cognitiva, $25,3 \%$ psicomotora e $7,5 \%$ afetiva (Figura 3).

Foram registradas, em média, oito questões por ciclo e, no período avaliado (quatro semestres), foram realizados seis ciclos por semestre. De acordo com a taxonomia de Bloom, 32 questões foram consideradas de baixa taxonomia (1), 22 de média (2) e 13 de alta taxonomia (3), conforme a Figura 4.

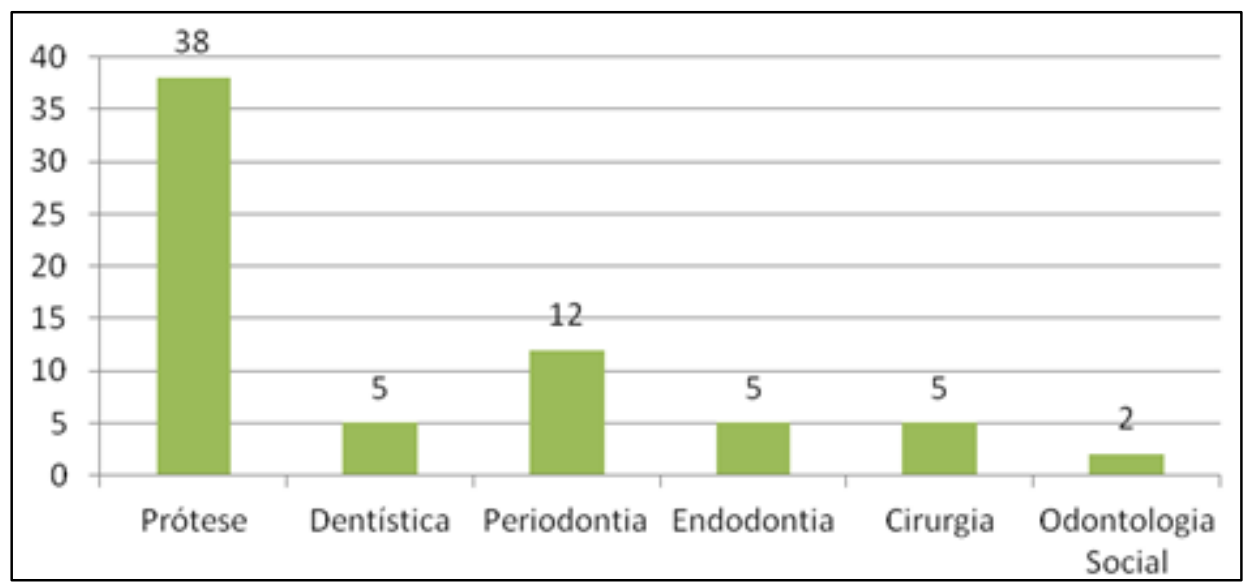

Figura 2. Distribuição das questões por área de conhecimento. 


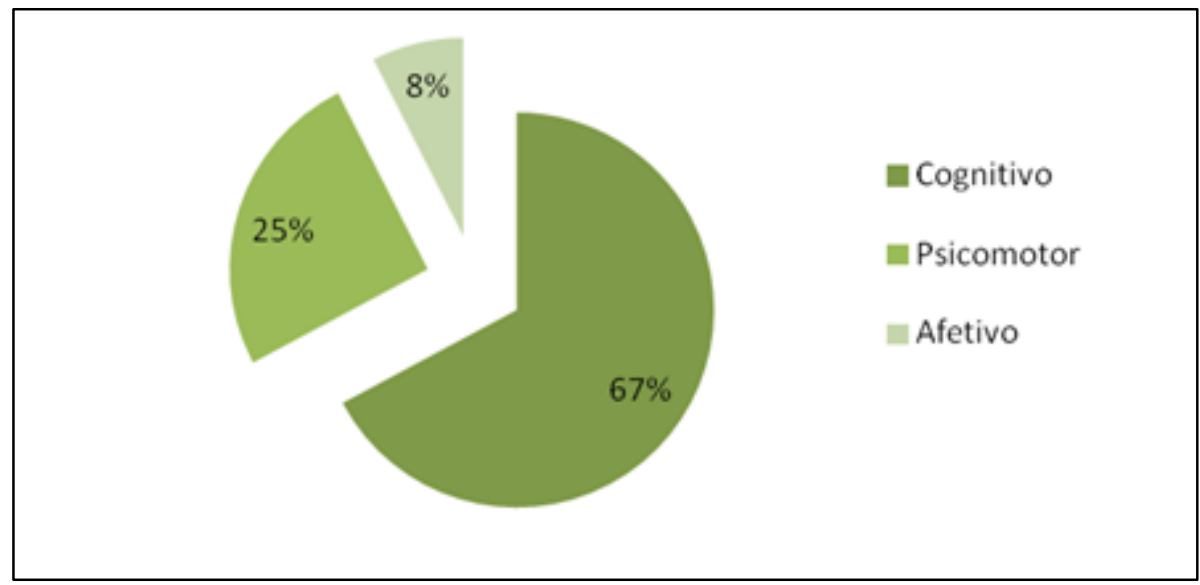

Figura 3. Distribuição das questões por eixos de competência profissional.

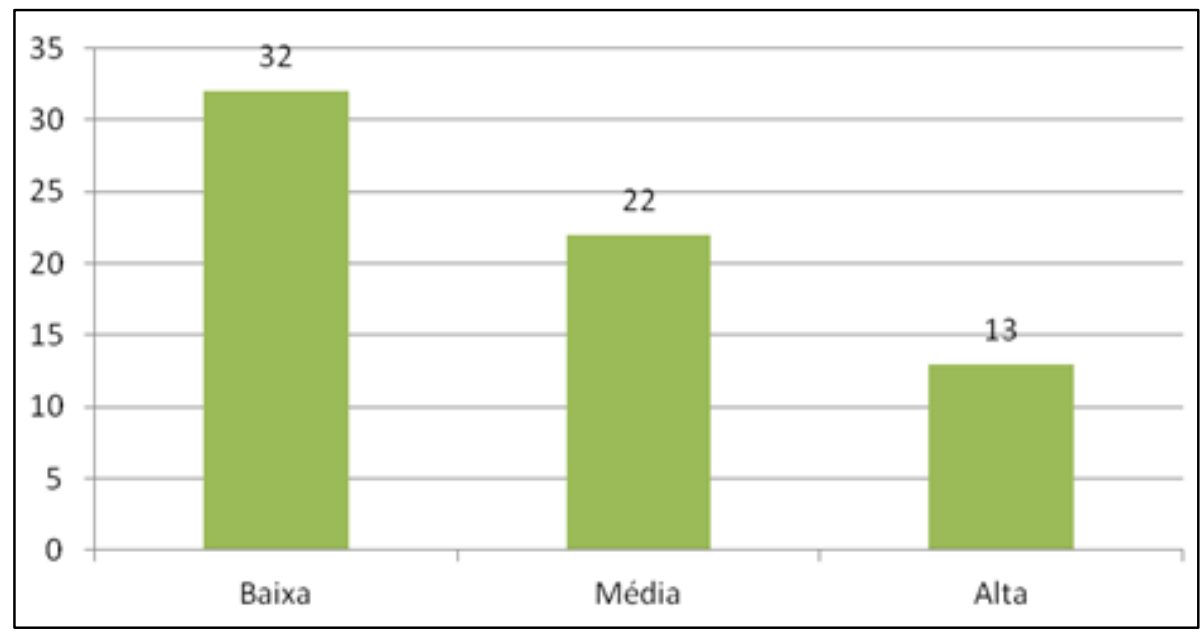

Figura 4. Classificação das questões pela Taxonomia de Bloom

\section{DISCUSSÃO}

A problematização como método ativo de ensino-aprendizagem pode ser observada neste acompanhamento de dois anos realizados na Faculdade de Odontologia da SUPREMA. O currículo do curso apresenta um modelo híbrido, com modalidades de ensino convencionais, combinadas com problematização para pequenos grupos ${ }^{11}$. Baseado no relato dos estudantes, percebe-se claramente que a metodologia incentivou a busca de informações e estimulou aprender a aprender, de acordo com o que é preconizado pelas Diretrizes Curriculares Nacionais ${ }^{4}$.

Neste contexto, a problematização através do ciclo pedagógico, como processo de aprendizagem, contribuiu para que o estudante pudesse compreender sua formação e, ainda, apontou inseguranças nos processos de trabalho. Das inseguranças, destacaram-se, principalmente, as relacionadas à especilidade prótese dentária, o que pode ser justificado, sobretudo, pelo maior grau de complexidade exigido neste momento da formação do estudante. Justifica-se, ainda, 
pela ausência de casos de prótese na rede pública (Sistema Único de Saúde - SUS), onde os estudantes realizam o estágio supervisionado. Apesar de a formação dos estudantes exigir a identificação das necessidades de saúde do paciente, os casos de prótese não são contemplados na rede pública, o que estimula o maior interesse no atendimento desses casos na clínica integrada.

Os cursos da área da Saúde estão estruturados sob um modelo pedagógico impregnado com uma visão fragmentada, para a formação tecnicista e para a especialidade $^{12}$. Esta afirmação vai de encontro à proposta pedagógica institucional da SUPREMA, mas, na prática, mesmo que o currículo contemple uma formação integrada, as atividades realizadas na rede pública sofrem uma influência da formação ainda fragmentada dos profissionais inseridos neste contexto.

Ao mesmo tempo, é natural que a maior complexidade exigida pelos procedimentos a serem realizados no último período de formação gerem maior insegurança ao estudante. Apesar de ser uma clínica integrada no sentido mais amplo, os resultados obtidos pelo método da problematização refletem uma maior resolução de casos clínicos que abordaram planejamento protético para reabilitação oral, mesmo que contemplando todas as especialidades.

Os resultados apresentados neste estudo demonstraram que as questões levantadas pelos estudantes durante o ciclo pedagógico dos diferentes semestres avaliados se repetiram, o que pode ser justificado pelo perfil de morbimortalidade da clínica, uma vez que as necessidades pré-protéticas dos pacientes já foram resolvidas nas clínicas integradas precedentes.

A atuação docente no ensino superior deve compreender os níveis presentes na taxonomia de Bloom, ficando claro que um mesmo conteúdo pode ser aprendido e ensinado com objetivos diferentes ${ }^{8}$. A atuação envolvendo os diferentes níveis de taxonomia possibilita que a relação de ensino e aprendizagem desenvolva nos alunos diferentes competências, visando sua atuação profissional em contexto multifacetado e multideterminado, que exige do indivíduo diferentes habilidades para a resolução de situações complexas. Por isso é de fundamental importância que os instrumentos de avaliação incorporem os diferentes níveis da taxonomia dos objetivos educacionais. Tal afirmação vai ao encontro dos resultados deste trabalho, deixando claro que o método da problematização compreende todos os níveis da taxonomia do domínio cognitivo.

De acordo com outros autores ${ }^{13}$, a educação deve organizar-se em torno de quatro pilares do conhecimento: aprender a conhecer (ou aprender a aprender, adquirir os instrumentos de compreensão); aprender a fazer (para poder agir sobre o meio envolvente); aprender a viver juntos (a fim de participar e cooperar com os outros em todas as atividades humanas); aprender a ser (via essencial que integra as três precedentes). Os resultados deste trabalho mostram que, apesar de as questões levantadas abordarem os quatro pilares do conhecimento, houve uma porcentagem baixa de questões de abordagem afetiva. As questões levantadas aproximam-se do perfil tecnicista do cirurgião dentista formado pelo currículo tradicional, valorizando o aprimoramento 
cognitivo e psicomotor, em detrimento ao afetivo.

Ao aplicar a metodologia da problematização na clínica integrada, percebeu-se a possibilidade de formar um estudante com uma postura mais ampla sobre as necessidades de saúde do paciente e não só focado na resolução técnica do caso. Associado a isso, notou-se que tal metodologia incentivou uma atitude mais ativa do estudante, permitindo desenvolver suas competências profissionais. Em estudo similar realizado na Universidade de Harvard ${ }^{14}$, os autores acrescentaram que esta formação favorece um melhor desempenho clínico e contribui para um melhor cuidado para com o paciente.

As fragilidades detectadas pelos resultados do estudo permitiram reflexões que geraram adequações no currículo, principalmente no que diz respeito ao critério de avaliação docente e discente, e também pela aplicação de metodologia ativa nos períodos iniciais do curso.

\section{CONCLUSÃO}

A partir dos resultados encontrados no presente estudo pode-se observar que, ao aplicar a problematização como método ativo de ensino-aprendizagem, surgiram, em sua maioria, questões de abordagem cognitiva e de baixa taxonomia de Bloom.

\section{REFERÊNCIAS}

1. Feuerwerke LCM. Educação dos profissionais de saúde hoje: problemas, desafios, perpectivas e as propostas do Ministério da Saúde. Rev Abeno. 2003;3(1):24-7.

2. Conselho Nacional de Educação. Câmara de Educação Superior. Resolução CNE/CES no 3, de 19 de fevereiro de 2002. Institui Diretrizes Curriculares Nacionais do Curso de Graduação em Odontologia. Disponível em: http://portal.mec. gov.br/cne/arquivos/pdf/CES032002 .pdf.

3. Ribeiro DM, Rauen MS, Prado ML. $\mathrm{O}$ uso da metodologia problematizadora no ensino em Odontologia. Rev Odonto. Uni. Cid. São Paulo. 2007;19(2):217-21.

4. Aguilar-da-Silva RH, Miguel SS, Teixeira LS. Problematização como método ativo de ensinoaprendizagem: estudantes de farmácia em cenários de prática. Trab Educ Saúde. 2011, 9 (1): 77-93.

5. Aguilar-da-Silva RH, Perim GL, Abdalla IG et al. Abordagens pedagógicas e tendências de mudanças nas escolas médicas. Rev Bras Educ Med 2009:33(1 supl 1):53-62.

6. Bordenave JD, Pereira AM. Estratégias de ensino-aprendizagem. Petrópolis: Vozes, 1991.

7. Bloom BS. Taxonomy of Educational Objectives, Handbook I: The Cognitive Domain. New York: David McKay Co Inc.,1956.

8. Pelissoni AMS. Objetivos educacionais e avaliação da aprendizagem. Anuário Prod Acad Docente. 2009:3(5):129-39.

9. $\mathrm{Su}$ WM, Osisek PJ. The revised Bloom's Taxonomy: implications for educating nurses. J Contin Educ Nurs 2011 42(7):321-7.

10. Waal P, Telles M. A Taxonomia de Bloom. Dynamiclab. 2004. Disponível em: http://pt.scribd. com/doc/72836415/A-Taxonomia- 
de-Bloom-Waal-e-Telles.

11. Callis AN, McCann AL, Schneiderman ED et al. Application of basic science to clinical problems: traditional vs. hybrid problem-based learning. J Dent Educ. 2010;74(10):1113-24.

12. Ivama AM, Batista CVM, Silva RMR. Repensando os estágios. Londrina: Olho Mágico, 1997.

13. Delors J, Al-Mufti I, Amagi I, Carneiro R, Chung F, Geremek B et al. Educação: um tesouro a descobrir. Relatório para Unesco da Comissão Internacional sobre educação para o século XXI. 4 ed. São Paulo: Cortez, 2000.

14. Park SE, Susarla HK, Nalliah R, Timothé P, Howell TH, Karimbuz NY. Does a case completion curriculum influence dental students' clinical productivity? J Dent Educ. 2012;76(5):602-8.

\section{ABSTRACT \\ Problem based learning as an active method for teaching-learning in a Dental Course}

The National Curriculum Guidelines for Dentistry courses launched in 2002 indicated a broader health concept and identified a need for an active role of students in the teaching-learning process, proposing a shift of emphasis from content to more active and independent participation, and overcoming the dichotomy between theory and practice, emphasizing joint work with health services and populations. This study aimed to verify the effect of questioning on performance, for constructing questions related learning as a function of the knowledge area, competence axes, and Bloom's taxonomy of questions. Over 4 semesters we evaluated 93 students. From patient care experiences in the clinic, knowledge gaps were raised in the pedagogical cycle, which applied the methodology of questioning. Following tutorial steps, 182 questions were recorded. Of these, 67 were not repeated and were grouped by area of knowledge $(67.2 \%$ for cognitive, $25.3 \%$ for psychomotor and $7.5 \%$ for affective approach). According to Bloom's taxonomy, 32 questions were considered low taxonomy, 22 medium and 13 high taxonomy. From the results of this study it can be concluded that by applying questioning as an active method of teaching and learning, mostly issues of cognitive approach and low Bloom's taxonomy have emerged.

Descriptors: Problem-based Learning. Health Education. Educational

Assessment.

Correspondência para:

Antônio Márcio Lima Ferraz Júnior

e-mail: amlfj@yahoo.com.br

Faculdade de Ciências Médicas e da Saúde de Juiz de Fora - SUPREMA

Alameda Salvaterra $n^{\circ} 200$

36033-003 Salvaterra - Juiz de Fora, MG 